[7] A. Hafeez and W. E. Stark, "Decision feedback sequence estimation for unwhitened ISI channels with applications to multiuser detection," IEEE J. Select. Areas Commun., vol. 16, pp. 1785-1795, Dec. 1998.

[8] W. H. Gerstacker, F. Obernosterer, R. Meyer, and J. B. Huber, "On prefilter computation for reduced-state equalization," IEEE Trans. Wireless Commun., vol. 1, pp. 793-800, Oct. 2002.

[9] W. H. Gerstacker and R. Schober, "Equalization concepts for EDGE," IEEE Trans. Wireless Commun., vol. 1, pp. 190-199, Jan. 2002.

[10] G. D. Forney Jr, "Maximum-likelihood sequence estimation of digital sequences in the presence of intersymbol interference," IEEE Trans. Inform. Theory, vol. IT-18, pp. 363-378, Mar. 1972.

[11] G. Ungerböck, "Adaptive maximum-likelihood receiver for carrier-modulated data-transmission systems," IEEE Trans. Commun, vol. COM-22, pp. 624-636, May 1974.

[12] M. Magarini, A. Spalvieri, and G. Tartara, "The mean-square delayed decision feedback sequence detector," IEEE Trans. Commun., vol. 50, pp. 1462-1470, Sept. 2002.

[13] M. V. Eyuboglu and S. U. Qureshi, "Reduced-state sequence estimation with set partitioning and secision feedback," IEEE Trans. Commun., vol. COM-36, pp. 13-20, Jan. 1988

[14] P. Höher, "TCM on frequency-selective fading channels: A comparison of soft-output probabilistic equalizers," in Proc. IEEE Global Telecommunication Conf. (GLOBECOM'90), San Diego, CA, Dec. 1990, pp. 401.4.1-401.4.6.

[15] W. Koch and A. Baier, "Optimum and sub-optimum detection of coded data disturbed by time-varying intersymbol interference," in Proc. IEEE Global Telecommunication Conf. (GLOBECOM'90), San Diego, CA, Dec. 1990, pp. 807.5.1-807.5.6.

[16] A. Hafeez and W. E. Stark, "Soft-output multiuser estimation for asynchronous CDMA channels," in Proc. IEEE Vehicular Technology Conf. (VTC'97), Phoenix, May 1997, pp. 465-469.

[17] GSM Recommendation 05.05: "Propagation Conditions," Vers. 5.3.0 Release, 1996.

[18] P. Jung, "Laurent's representation of binary continuous phase modulated signals with modulation index $1 / 2$ revisited," IEEE Trans. Commun., vol. 42, pp. 221-224, Feb./Mar./Apr. 1994.

\section{Reduced Complexity I/Q Turbo Detection for Space-Time Trellis-Coded Systems}

\author{
Bee Leong Yeap and Lajos Hanzo
}

\begin{abstract}
A reduced-complexity turbo-detection scheme, referred to here as the R-TD arrangement, is proposed for employment in space-time trellis-coded (STTC) systems using the inphase/quadrature-phase (I/Q) cancellation technique that was previously developed for single-transmitter and single-receiver systems. The R-TD scheme decomposes the received signal into its constituent $I$ and $Q$ signal components and detects these components separately; hence, reducing the number of possible signal combinations to be "tested" by the detector. The R-TD scheme is capable of approaching the performance of the conventional turbo detector (F-TD), while achieving a complexity reduction factor of 3 and 862 for a four-phase-shift-keying 32-state STTC system, denoted as STTC $(4,32)$, communicating over two- and five-path Rayleigh-fading channels, respectively, exhibiting a symbol-spaced and equal-tap-weight channel-impulse response. In order to investigate the benefits of employing channel coding in conjunction with STTC schemes, the R-TD principle was also invoked in convolutional-coding-aided STTC schemes. It was observed that, at a given throughput, the turbo-detected "nonchannel-coded" STTC $(4,32)$ system required a similar signal-to-noise ratio to that of the $R=1 / 2$ and constraint length $K=7$ convolutional-coded STTC $(16,16)$ scheme for achieving the bit error rate (BER) of $10^{-5}$. At a higher target, BER of $10^{-3}$, the "nonchannel-coded" STTC $(4,16)$ and STTC $(4,32)$ schemes outperformed the channel-coded STTC $(16,16)$ system by 0.8 and $2.3 \mathrm{~dB}$, despite having a factor of 19 or 11 lower complexity, respectively.
\end{abstract}

Index Terms-Inphase, iterative equalization and decoding, quadrature phase, reduced complexity, serial concatenated codes, space-time trellis coding (STTC), turbo equalization/detection.

\section{INTRODUCTION}

In the third-generation (3G) mobile radio standard [1], a transmit diversity option has been proposed for increasing the link quality and, therefore, the capacity of the system. Spatial diversity may be achieved with the aid of multiple transmitters or receivers. Often, it is more cost effective to employ the multiple antennas at the base station, instead of upgrading millions of handsets. Recently, attractive transmit diversity techniques known as space-time block coding (STBC) [2]-[4] and space-time trellis coding (STTC) [4], [5] have been advocated for providing additional diversity gains, as well as for overcoming the limited capacity offered by the hostile fading wireless channel [4]. STBCs do not provide coding gain unless concatenated with an outer channel code. The main benefit of STBCs is the provision of full diversity with the aid of a simple decoding scheme. Hassibi and Hochwald [6] proposed a different transmit diversity scheme, referred to as a linear dispersion (LD) code, which also requires simple decoding while maximizing the mutual information between the transmit and receive signals. In contrast to STBCs, STTC relies on the joint design of channel coding, modulation, and transmit diversity, as well as optional receiver-diversity schemes. The design rules proposed in [5] resulted in maximum-diversity gain for systems employing two transmit antennas. Subsequently, systematic transmit-diversity design methods were developed in [7] and [8] for an arbitrary number of transmit antennas. These transmit

Manuscript received February 12, 2003; revised December 18, 2003 and February 20,2004. This work has been supported in the framework of the pan-European project SCOUT, which is funded in part by the European Union.

The authors are with the School of Electrical and Computer Science, University of Southampton, Southampton Hants SO17 1BJ, U.K. (e-mail: bly@ecs.soton.ac.uk; 1h@ecs.soton.ac.uk).

Digital Object Identifier 10.1109/TVT.2004.830970 


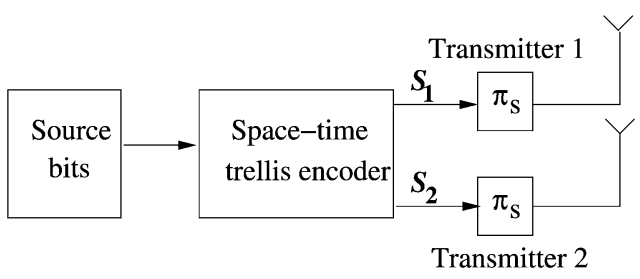

Fig. 1. Transmitter of the STTC system, where $\pi_{s}$ represents the space-time trellis code's interleaver.

diversity schemes may be employed in conjunction with orthogonal frequency-division multiplexing (OFDM) systems [9] in order to mitigate the effects of frequency-selective channels.

Typically, detection of the STTC symbols is constituted by independent channel equalization and space-time trellis decoding operations. However, instead of implementing the channel-equalization and STTC-decoding operations independently, the performance of the STTC system can be further improved by performing both operations iteratively. This philosophy is based on that of turbo equalization [10], which was first employed in a convolutional-encoded binary phase-shift keying (BPSK) system transmitting over dispersive channels. This technique performs channel equalization and channel decoding iteratively and has been shown to successfully mitigate the effects of channel intersymbol interference (ISI) [4]. Bauch et al. [11] adapted the turbo-equalization technique for iterative channel equalization and STTC decoding. This approach yielded an improved performance owing to the intelligent exploitation of the soft-decision-based feedback provided by the STTC decoder output for the equalizer.

In [4] and [12], the performance of the STTC system was improved by employing additional channel encoding and turbo detection. However, owing to the high complexity of the turbo detector, the investigations were limited to channel-impulse responses (CIR) associated with low dispersions. In this paper, we propose a reduced-complexity turbo detector designed for STTC systems employing convolutional channel coding or no channel coding, which we refer to as the CC-STTC and NOCC-STTC schemes, respectively. Initially, the performance of these systems is analyzed in isolation and is assessed in terms of the achievable bit error rate (BER) performance and computational complexity. The impact of the tolerable system delay on the performance of the proposed turbo-detection technique is also investigated, which is predominantly determined by the length of the channel interleavers utilized. Subsequently, we will compare the performance of the CC-STTC scheme, which is an $R=1 / 2$ channel-coded 16-state 16QAM system, to that of the 4PSK STTC benchmarker refraining from using channel coding, while maintaining the same effective throughput. The aim of this comparison is to investigate whether the achievable BER performance benefits outweigh the associated computational cost and the reduction in effective throughput.

We commence our discourse by presenting an overview of the investigated system in Section II. This is followed by an overview of the reduced-complexity turbo-detection principles and a complexity analysis in Sections III and IV, respectively. Section V presents our simulation results and, finally, we conclude our discourse in Section VI.

\section{SYSTEM OVERVIEW}

In our investigation, we have employed two different STTC systems. The first scheme is the NOCC-STTC arrangement, shown in Fig. 1, which consists of an STTC encoder using $T_{x}=2$ transmit antennas. In the second STTC system, which we earlier referred to as the CC-STTC.
A rate $R=1 / 2$ convolutional encoder is concatenated with a twotransmitter STTC encoder, as shown in Fig. 2.

Let us commence by describing the NOCC-STTC scheme, which is followed by the portrayal of the CC-STTC system. For the NOCC-STTC scheme, the source bits are directly passed to the STTC encoder, represented by using the notation $\operatorname{STTC}(M, n)$, where $M$ denotes the modulation mode used and $n$ the number of states of the STTC encoder [5]. These STTC codes can be characterized by using the trellis diagrams seen in [5]. Upon receiving an input symbol formed from the source bits, the STTC produces an output STTC symbol in each transmitter arm of Fig. 1. These output symbols are referred to here as the STTC symbols, constituted by $S_{1}$ and $S_{2}$ in Fig. 1. At the output of the STTC encoder, the encoded symbols are interleaved by a random STTC symbol interleaver represented as $\pi_{s}$ in Fig. 1. Note that the same interleaving rule is used for all transmit antennas [5].

For the CC-STTC system, the source bits are first passed to a channel encoder, which subsequently directs the interleaved encoded bits to a two-transmitter STTC encoder. Two different channel encoders were employed, namely, a 1/2-rate convolutional code having a constraint length of $K=5$ and octal generator polynomials of $G_{0}=35$ and $G_{1}=23$, as well as a $1 / 2$-rate $K=7$ convolutional code using $G_{0}=$ 133 and $G_{1}=171$ [13] octal generator polynomials. At the output of the STTC encoder, the encoded symbols are interleaved by a random STTC symbol interleaver represented as $\pi_{s}$ in Fig. 2. Note that random interleavers are utilized for both the bit-based channel interleaver and for the symbol-based STTC interleaver. As mentioned before, the same interleaving rule is used for all transmit antennas in order to preserve the rank property of the space-time codes [5].

In our investigations, we employed a time-division multiple-access/time-division-duplex (TDMA/TDD) system, which has eight uplink and eight downlink slots and where one transmission slot will be available after every 16 TDMA slots. Each transmission burst has a duration of $72 \mu \mathrm{s}$ and consists of 144 data symbols. Additionally, two- and five-path Rayleigh-fading channels having equal symbol-spaced tap weights were used. The Rayleigh-fading statistics obeyed a normalized Doppler frequency of $3.3165 \times 10^{-5}$, where the fading magnitude and phase was kept constant for the duration of a transmission burst. The normalized Doppler frequency is based on a transmission rate of $2600 \mathrm{KBaud}$, a transmission frequency of $1.9 \mathrm{GHz}$, and a vehicular speed of $30 \mathrm{mph}$. Furthermore, in order to characterize the best possible performance of these systems, the CIR was perfectly estimated at the receiver. As shown in Fig. 1, a pair of $R_{x}=2$ receive antennas was employed at the receiver. Turbo detection was then used, whereby the channel-equalization space-time trellis and channel decoding (only for the CC-STTC system) operations were performed iteratively. The received signal at the $i$ th receiver can be written as

$$
\begin{aligned}
r^{i}(t) & =\sum_{k=1}^{T_{x}=2} s^{k}(t) * h^{k i}(t)+n^{i}(t) \quad i=1 \ldots R_{x}=2 \\
& =r_{I}^{i}(t)+j r_{Q}^{i}(t)
\end{aligned}
$$

where $s^{k}(t), r^{i}(t)$, and $n^{i}(t)$ denote the symbol transmitted from the $k$ th transmitter, the received signal of the $i$ th receive antenna, and the additive white Gaussian noise (AWGN) imposed on the signal received by the $i$ th receive antenna, respectively. The notation $h^{k i}(t)$ refers to the CIR corresponding to the $k$ th transmit and $i$ th receive link, whereas * represents convolution. Additionally, the subscripts $I$ and $Q$ represent the inphase and quadrature-phase components, respectively. The signal-to-noise ratio (SNR) is defined as the ratio of the average received power to the noise power per received antenna. Since the average 


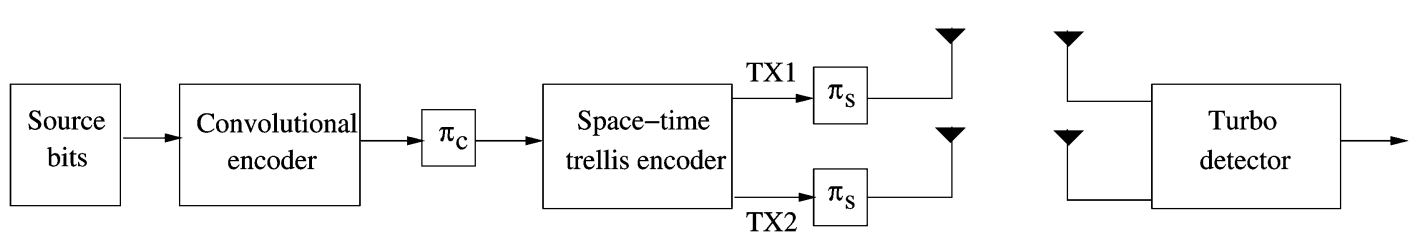

Fig. 2. Schematic of the STTC system employing turbo detection at the receiver. The notation $\pi_{s}$ represents the space-time trellis code's interleaver.

received power is equal to the total average transmitted power, the SNR is $E\left[\left\|s^{k}(t)\right\|^{2}\right] / \sigma^{2}$, where $\sigma^{2}$ is the variance of the noise component $n^{i}(t)$.

\section{PRINCIPLES OF REDUCED-COMPLEXITY I/Q TURBo DETECTION}

After transmission over a channel having complex-valued CIR, the received I/Q signals in (1), namely, $r_{I}^{i}(t)$ and $r_{Q}^{i}(t)$, become dependent on the inphase and quadrature-phase components of the signal $s_{k}(t)$, namely, on $s_{I}^{k}(t)$ and $s_{Q}^{k}(t)$, respectively. This interdependency between $s_{I}^{k}(t)$ and $s_{Q}^{k}(t)$ in the received quadrature signals $r_{I}^{i}(t)$ and $r_{Q}^{i}(t)$ is referred to here as cross-coupling.

As a consequence of the cross-coupling, the receiver has to consider an increased number of signal combinations for identifying the most likely transmitted symbol, hence necessitating a high number of equalizer trellis states. However, the number of trellis states to be considered can be reduced significantly when the cross-coupling is removed such that the quadrature components of the decoupled channel output $r^{i^{\prime}}(t)$, which consists of $r_{I}^{i^{\prime}}(t)$ and $r_{Q}^{i^{\prime}}(t)$, are only dependent on $s_{I}^{k}(t)$ or $s_{Q}^{k}(t)$. This is the basic principle behind the reduced-complexity turbo-detection scheme.

In order to perform the decoupling, the quadrature components of the signal estimates, namely, $\hat{s}_{I}^{k}(t)$ and $\hat{s}_{Q}^{k}(t)$ of all transmitters, are generated by averaging the weighted a posteriori probabilities of the bits corresponding to each quadrature arm, as demonstrated in [4], [14], and [15]. Subsequently, these estimated symbols are convolved with the inphase and quadrature-phase CIR estimates of the $k$ th transmit and $i$ th receive link, in order to generate $\hat{s}_{I}^{k}(t) * \hat{h}_{I}^{k i}(t), j \hat{s}_{I}^{k}(t) * \hat{h}_{Q}^{k i}(t)$, $\hat{s}_{Q}^{k}(t) * \hat{h}_{Q}^{k i}(t)$, and $j \hat{s}_{Q}^{k}(t) * \hat{h}_{I}^{k i}(t)$, where $k=1 \ldots T_{x}$ and $i=$ $1 \ldots R_{x}$. With the aid of these signals, the cross-coupling between the received quadrature signals can be removed using the procedures outlined in [4], such that we obtain the decoupled received signals $r_{I}^{i^{\prime}}(t)$ and $r_{Q}^{i^{\prime}}(t)$, which are only dependent on a particular quadrature component, namely, on $s_{I}^{k}(t)$ or $s_{Q}^{k}(t)$, rather than on both. For example, the signal $r_{I}^{i^{\prime}}(t)$ can be generated by removing $\hat{s}_{Q}^{k}(t) * \hat{h}_{Q}^{k i}(t)$ and $j \hat{s}_{Q}^{k}(t) * \hat{h}_{I}^{k i}(t)$ from the received signal $r^{i}(t)$, yielding

$$
\begin{aligned}
r_{I}^{i^{\prime}}(t)= & r^{i}(t)+\sum_{k=1}^{T_{x}=2}\left(\hat{s}_{Q}^{k}(t) * \hat{h}_{Q}^{k i}(t)-j \hat{s}_{Q}^{k}(t) * \hat{h}_{I}^{k i}(t)\right) \\
= & \sum_{k=1}^{T_{x}=2}\left(s_{I}^{k}(t) * h_{I}^{k i}(t)+j s_{I}^{k}(t) * h_{Q}^{k i}(t)\right. \\
& \left.\quad+e\left(\hat{s}_{Q}^{k}(t), \hat{h}_{Q}^{k i}(t)\right)+j e\left(\hat{s}_{Q}^{k}(t), \hat{h}_{I}^{k i}(t)\right)\right) \\
& +n^{i}(t)
\end{aligned}
$$

where $e\left(\hat{s}_{Q}(t), \hat{h}_{Q}(t)\right)$ and $e\left(\hat{s}_{Q}(t), \hat{h}_{I}(t)\right)$ are the error terms expressed as

$$
\begin{aligned}
& e\left(\hat{s}_{Q}^{k}(t), \hat{h}_{Q}^{k i}(t)\right)=r_{I}^{i}(t)+\hat{s}_{Q}^{k}(t) * \hat{h}_{Q}^{k i}(t) \\
& e\left(\hat{s}_{Q}^{k}(t), \hat{h}_{I}^{k i}(t)\right)=r_{Q}^{i}(t)-\hat{s}_{Q}^{k}(t) * \hat{h}_{I}^{k i}(t)
\end{aligned}
$$

which arise when inaccurate CIR estimates and low-confidence symbol estimates are generated. Similarly, $r_{Q}^{i^{\prime}}(t)$ is obtained by subtracting
$\hat{s}_{I}^{k}(t) * \hat{h}_{I}^{k i}(t)$ and $j \hat{s}_{I}^{k}(t) * \hat{h}_{Q}^{k i}(t)$, where $k=1 \ldots T_{x}$ and $i=1 \ldots R_{x}$ from $r^{i}(t)$. Although—no doubt-errors may be introduced in the decoupling operation, it was shown in [4] and [15] that the imperfect decoupling effects are gradually compensated through the successive turbo-detection iterations and the achievable performance approaches that of the turbo detector utilizing the conventional full-complexity trellis-based equalizer.

Having highlighted the philosophy of the decoupling operation, we continue by describing the structure of the turbo detector contrived for the lower-complexity NOCC-STTC system, which incorporates the I/Q decoupling principle. This is then followed by the description of the I/Q turbo detector designed for the more complex CC-STTC scheme. In the first turbo-detection iteration of Fig. 3, a multivariable decision feedback equalizer (MV-DFE) [16] was utilized. The MV-DFE was employed, since it constituted a low-complexity approach to providing initial estimates of the transmitted symbols, as compared to the more complex trellis-based equalizers. These soft decisions are passed to the STTC decoder of Fig. 3, which subsequently generates the a posteriori information. The extrinsic information is extracted from the a posteriori information and is directed to the I/Q equalizers. As mentioned previously, the a posteriori probability is utilized in the symbol-regeneration process, which aids the decoupling operation, whereas the extrinsic information becomes the a priori information employed by the I/Q equalizer in the second turbo-detection iteration. The decoupling is then performed using the regenerated symbols and with the aid of (2). After the decoupling operation has been completed, the resultant signals corresponding to the inphase signal components $r_{I}^{i^{\prime}}(t)$ of all transmitters are directed to an I/Q equalizer, while the quadrature-phase signal components $r_{Q}^{i^{\prime}}(t)$ of all transmitters are invoked by the second I/Q equalizer. The logarithmic likelihood ratio (LLR) values generated by the I/Q equalizers of Fig. 3 are deinterleaved by the STTC deinterleaver and passed to the STTC decoder. The STTC decoder computes the a posteriori LLRs, which are then processed for generating the extrinsic information. As in the first iteration, the a posteriori LLR is used for symbol regeneration, while the extrinsic LLR becomes the $a$ priori LLR for the I/Q equalizers in the next turbo-detection iteration. This iterative process is repeated until the termination criterion stipulated is satisfied. In our investigations, the iterations were curtailed when no significant further performance gains could be obtained through additional iterations. Both the I/Q decoupling operations and the reduced complexity space-time trellis turbo-equalization algorithm is summarized in Fig. 4.

In the case of the more complex CC-STTC system, the structure of the R-TD arrangement is similar to that of the lower complexity NOCC-STTC R-TD scheme seen in Fig. 2, with the exception that the STTC decoder does not directly pass the computed extrinsic information to the I/Q equalizers. Instead, the extrinsic information is deinterleaved by a channel deinterleaver and passed to the channel decoder. The channel decoder processes this information in order to generate the a posteriori LLR values. Subsequently, the extrinsic LLR is once again extracted from the a posteriori LLRs computed by the channel decoder and directed back to the I/Q equalizer.

In our investigations, we have utilized the max-log-maximum $a$ posteriori (MAP) algorithm [17], [18] in our I/Q equalizers, the conventional channel equalizer, and the STTC decoder, as well as in the convo- 


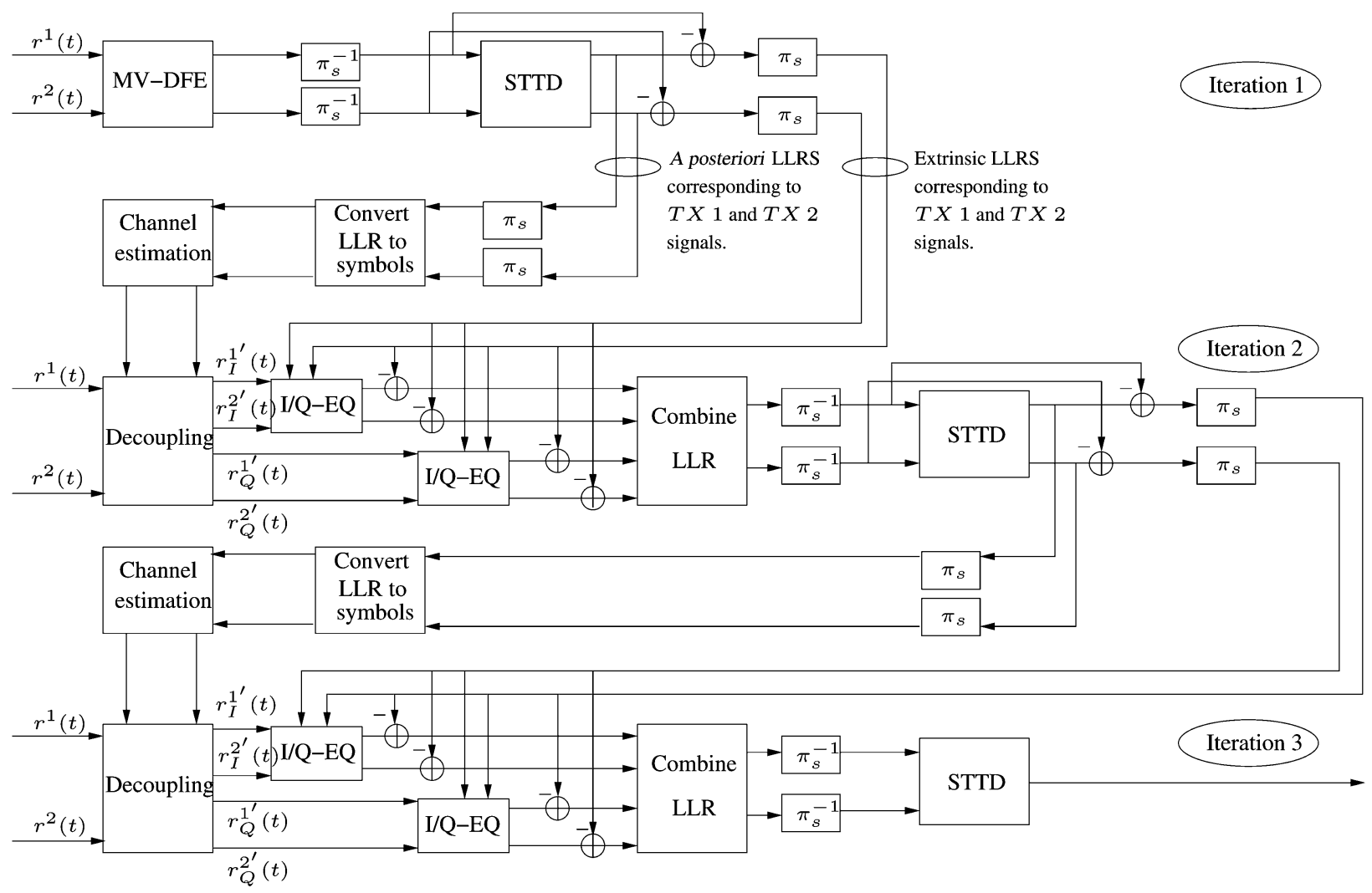

Fig. 3. Schematic of the reduced-complexity turbo detector in conjunction with a two-transmitter-two-receiver-based STTC scheme performing three turbo-detection iterations.

lutional decoder, since it constitutes a good compromise in terms of the achievable performance and the computational complexity imposed.

\section{COMPLEXITY ANALYSIS}

For simplicity, the complexity of the turbo detectors can be quantified in terms of the number of associated trellis transitions per information bit. Therefore, the complexity of the equalizer and STTC decoder, which is dependent on the number of trellis transitions per symbol, must be normalized by the number of original information bits per symbol $T_{r}$, hence yielding the number of transitions per information bit.

For the conventional full-complexity trellis-based STTC channel equalizer [4], termed as CT-EQ, it can be shown that the complexity $\Lambda$ CT-EQ], associated with equalizing the STTC signals transmitted over a channel having complex CIR tap weights and a delay spread of $\tau_{d}$ symbols, is

$$
\begin{aligned}
\Lambda[\mathrm{CT}-\mathrm{EQ}] & =\frac{\text { (Number of states } \cdot \text { Number of transitions) }}{T_{r}} \\
& =\frac{\left(M^{T_{x} \tau_{d}} \cdot M^{T_{x}}\right)}{T_{r}}=\frac{\left(M^{T_{x}\left(\tau_{d}+1\right)}\right)}{T_{r}}
\end{aligned}
$$

where, again, $M, T_{x}$, and $T_{r}$ denote the number of constellation points, the number of transmitters, and the number of information bits per symbol, respectively. By contrast, the complexity of a single I/Q equalizer (I/Q-EQ) trellis stage is given by

$$
\begin{aligned}
\Lambda\left[\frac{\mathrm{I}}{\mathrm{Q}-\mathrm{EQ}}\right] & =\frac{\text { (Number of states } \cdot \text { Number of transitions) }}{T_{r}} \\
& =\frac{\left(\sqrt{M}^{T_{x}\left(\tau_{d}+1\right)}\right)}{T_{r}} .
\end{aligned}
$$

For the $\operatorname{STTC}(M, n)$ decoder, where $M$ and $n$ denote the number of constellation points and the number of trellis states in the encoder, the complexity $\Lambda[\operatorname{STTC}(M, n)]$ incurred is

$$
\Lambda[\operatorname{STTC}(M, n)]=\frac{(n \cdot M)}{T_{r}} .
$$

Finally, the corresponding complexity of the $R=1 / 2$ and constraint length $K$ channel decoder $\Lambda[\mathrm{CC}]$ can be written as

$$
\Lambda[\mathrm{CC}]=2^{K} .
$$

Having determined the complexity of the channel equalizer, the STTC decoder, and the convolutional channel decoder as a function of the number of trellis transitions per information bit, the complexity of the F-TD in the context of the NOCC-STTC and CC-STTC systems can be estimated as

$$
\Lambda^{\mathrm{NOCC}-\mathrm{STTC}}[\mathrm{F}-\mathrm{TD}]=\frac{\left(\left(M^{T_{x}\left(\tau_{d}+1\right)}\right)+n \cdot M\right)}{T_{r} \operatorname{Itr}^{\mathrm{NOCC}-\mathrm{STTC}}[\mathrm{F}-\mathrm{TD}]}
$$

and

$$
\begin{aligned}
& \Lambda^{\mathrm{CC}-\mathrm{STTC}}[\mathrm{F}-\mathrm{TD}]=\left(\frac{\left(\left(M^{T_{x}\left(\tau_{d}+1\right)}\right)+n \cdot M\right)}{T_{r}}+2^{K}\right) \\
& \cdot \operatorname{Itr}^{\mathrm{CC}-\mathrm{STTC}}[\mathrm{F}-\mathrm{TD}]
\end{aligned}
$$




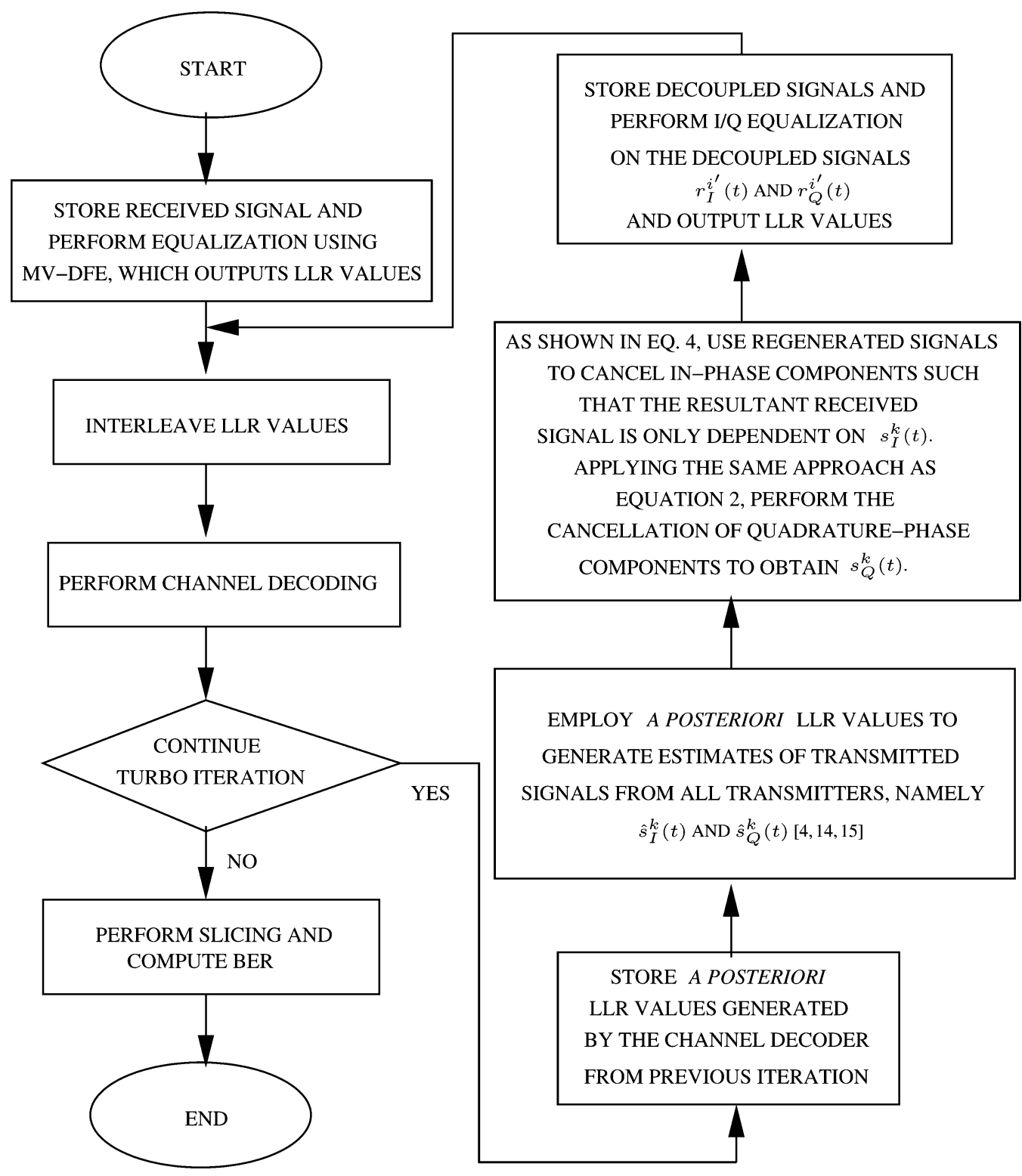

Fig. 4. Reduced-complexity space-time trellis turbo-equalization algorithm and summary of I/Q decoupling operations.

respectively. Finally, the complexity of the R-TD technique, studied in the context of the NOCC-STTC and CC-STTC systems, can be written as

$$
\begin{aligned}
& \Lambda^{\mathrm{NOCC}-\mathrm{STTC}}[\mathrm{R}-\mathrm{TD}]=\frac{n \cdot M}{T_{r}} \\
& +\frac{\left(2 \cdot\left(\sqrt{M}^{T_{x}\left(\tau_{d}+1\right)}\right)+n \cdot M\right)}{T_{r}} \\
& \times\left(\mathrm{Itr}^{\mathrm{NOCC}-\mathrm{STTC}}[R-T D]-1\right)
\end{aligned}
$$

and

$$
\begin{aligned}
\Lambda^{\left.\mathrm{CC}-\mathrm{STTC}_{[\mathrm{R}} \mathrm{TD}\right]=} & \left(\frac{n \cdot M}{T_{r}}+2^{K}\right) \\
& +\left(\frac{2 \cdot \sqrt{M}^{T_{x}\left(\tau_{d}+1\right)}+n \cdot M}{T_{r}}+2^{K}\right) \\
& \times\left(\mathrm{Itr}^{\mathrm{CC}-\mathrm{STTC}}[\mathrm{R}-\mathrm{TD}]-1\right)
\end{aligned}
$$

respectively. The term $\operatorname{Itr}^{A}[]$ denotes the number of turbo detection iterations invoked in system "A." A factor of two was introduced in the term $2 \cdot \sqrt{M}^{T}{ }^{\left(\tau_{d}+1\right)}$, found in (10) and (11), since two I/Q-EQs are required for performing the equalization. In (10), the first term on the right-hand side represents the complexity incurred in the first R-TD iteration, where a MV-DFE and a STTC decoder were employed. Similarly, in (11), the terms within the first brackets on the right-hand side represent the complexity incurred in the first R-TD iteration, where the combination of a MV-DFE, an STTC decoder, and a convolutional decoder was employed. For the sake of simplicity, we have assumed that the complexity of the MV-DFE is negligible when compared to the complexity of the I/Q-EQ and CT-EQ. Therefore, in the first turbo-detection iteration, the complexity of the R-TD-assisted NOCC-STTC scheme is only dependent on the complexity of the STTC decoder, while that of the R-TD-aided CC-STTC system is dependent on the complexity of both the STTC decoder and that of the convolutional decoder. The remaining terms within the second brackets correspond to the complexity of the subsequent R-TD iterations. 


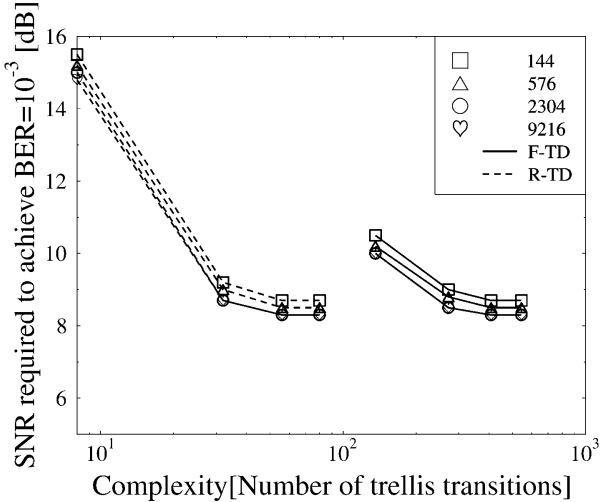

(a) NOCC-STTC $(4,4)$.

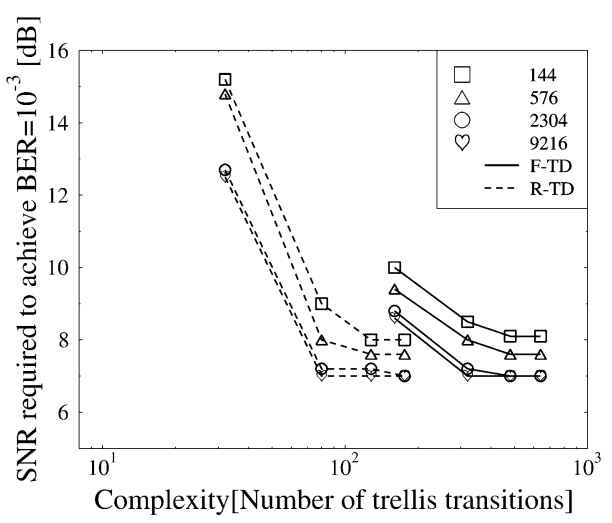

(c) NOCC-STTC $(4,16)$.

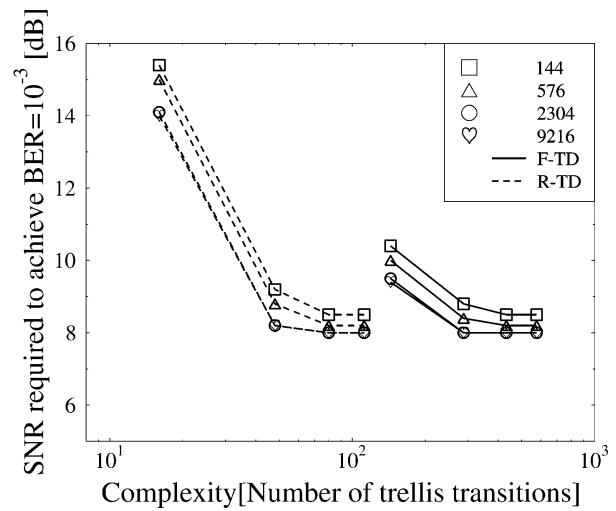

(b) NOCC-STTC $(4,8)$.

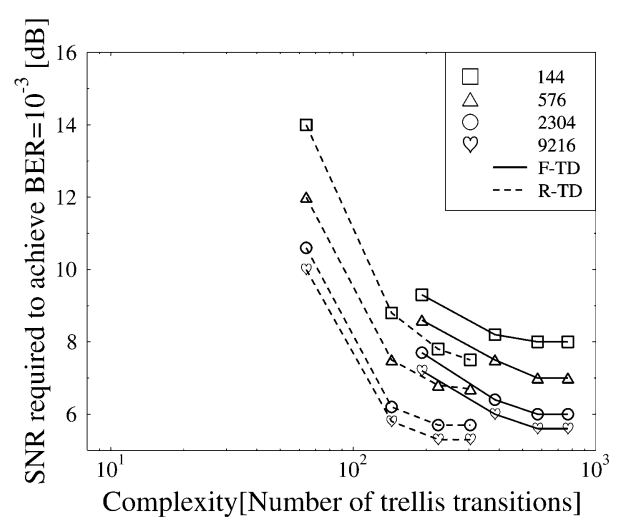

(d) NOCC-STTC $(4,32)$.

Fig. 5. Comparison of the reduced-complexity turbo detector and conventional turbo detector complexity for various nonchannel-coded STTC schemes employing interleaving lengths of 144, 576, 2304, and 9216 symbols. The signals were transmitted over a Rayleigh-fading channel exhibiting a two-path, equal-weight, and symbol-spaced CIR associated with a normalized Doppler frequency of $3.3615 \times 10^{-5}$. In each curve, the complexity increases upon increasing the number of turbo detection iterations. A maximum of four turbo-detection iterations were performed. The effective throughput was 2 bits/s.

\section{RESUltS AND DisCUSSIONS}

\section{A. BER Performance Versus Complexity for NOCC-STTC Systems}

Fig. 5 shows the BER performance versus complexity relationship for NOCC-STTC $(4, n)$ systems, where $n=4,8,16$ and 32 , using STTC interleavers of lengths $=144,576,2304$, and 9216 symbols. Note that we have chosen the BER of $10^{-3}$ for our comparisons, since this is often the target BER for wireless speech-transmission systems. Observe in Fig. 5 that, in order to achieve a BER of $10^{-3}$, the CC-STTC schemes using R-TD require similar SNR values to the F-TD technique, despite incurring a substantially lower computational complexity. More specifically, after three turbo-detection iterations, the SNR values required by the NOCC-STTC $(4,4)$, NOCC-STTC $(4,8)$, NOCC-STTC $(4,16)$, and NOCC-STTC $(4,32)$ systems employing $\mathrm{R}$-TD in order to achieve a BER of $10^{-3}$ were similar to those necessitated by the corresponding F-TD scheme performing two turbo-detection iterations, while achieving a complexity reduction factor of $9,6,4$, and 3 , respectively.

\section{B. Effect of STTC Interleaver Lengths on the Choice of Space-Time Trellis Codes}

Observe in Fig. 5 and Table I that for the NOCC-STTC systems utilizing a short STTC symbol interleaver length, i.e., the 144-symbol interleaver, the achievable performance gain of the different NOCC-STTC systems remained modest, ranging from 0.1 to 1.0 $\mathrm{dB}$, despite increasing the complexity of the STTC decoders owing to utilizing STTC encoders, which require more trellis states, such as the STTC $(4,32)$. In Fig. 5, when comparing the R-TD-aided performance
TABLE I

NONCHANNEL CODED STTC SYSTEMS, WHICH EMPLOY STTC SYMBOL-BASED INTERLEAVERS HAVING LENGTHS OF 144 , 576, 2304, AND 9216 SyMBOLS AND REDUCED-COMPLEXITY SPACE-TIME TRELLIS TURBo EQUALIZERS, INDICATING THE $E_{b} / N_{0}$ VALUES REQUIRED IN ORDER TO ACHIEVE A BER OF $10^{-3}$ IN COMPARISON TO THE STTC $(4,4)$ SYSTEM, I.E., THE RESULTS IN THE FIRST ROW OF THIS TABLE

\begin{tabular}{c|l|l|l|l}
\hline $\begin{array}{c}\text { NOCC-STTC systems using various } \\
\text { length symbol interleavers }\end{array}$ & $\begin{array}{l}144 \\
\text { symbols }\end{array}$ & $\begin{array}{l}576 \\
\text { symbols }\end{array}$ & $\begin{array}{l}2304 \\
\text { symbols }\end{array}$ & $\begin{array}{l}9216 \\
\text { symbols }\end{array}$ \\
\hline STTC $(4,4)$ & 8.5 & 8.5 & 8.5 & 8.4 \\
\hline STTC $(4,8)$ & 8.4 & 8.0 & 8.0 & 7.1 \\
\hline STTC $(4,16)$ & 8.0 & 7.5 & 7.0 & 6.8 \\
\hline STTC $(4,32)$ & 7.5 & 6.5 & 5.5 & 5.2 \\
\hline
\end{tabular}

of the NOCC-STTC $(4,4)$ system to that of the NOCC-STTC $(4,32)$ scheme using a 144-symbol interleaver after four turbo-detection iterations, it was observed that the NOCC-STTC $(4,32)$ scheme required a slightly lower SNR value, namely approximately $1.0 \mathrm{~dB}$ lower, in order to achieve the BER of $10^{-3}$. This was obtained at the cost of a factor of 4 higher decoding complexity. Similar trends were also observed for the F-TD scheme, where after four turbo-detection iterations the performance of the NOCC-STTC $(4,32)$ arrangement was observed to require a $1.0 \mathrm{~dB}$ lower SNR than the NOCC-STTC $(4,4)$, while incurring a factor of 1.4 higher complexity, in order to maintain BER $=10^{-3}$. Hence, for NOCC-STTC systems employing short 
interleaver lengths, such as the 144-symbol interleaver, it is sufficient to use the low-complexity $\operatorname{STTC}(4,4)$ code, since no significant performance gain is achieved by employing more complex and more powerful STTC codes. Further results of such comparisons is summarized are Table I.

However, for the more powerful NOCC-STTC schemes, such as the NOCC-STTC $(4,16)$ and the NOCC-STTC $(4,32)$ arrangements, it is observed that the NOCC-STTC system employing higher interleaver lengths requires lower SNR values for obtaining a BER of $10^{-3}$. For example, in Fig. 5(d), the STTC $(4,32)$ system utilizing the R-TD and using a 2304-symbol interleaver requires an SNR of $5.6 \mathrm{~dB}$ for achieving $\mathrm{BER}=10^{-3}$, whereas the same system employing the 144-symbol interleaver needs $7.5 \mathrm{~dB}$ to attain the same BER. Similarly, for the NOCC-STTC $(4,16)$ and NOCC-STTC $(4,32)$ systems using F-TD, it is also observed that the systems employing the 2304-symbol STTC interleaver require 1.1-2.4 dB lower SNRs than the $\operatorname{STTC}(4,16)$ and $\operatorname{STTC}(4,32)$ systems using a 144 -symbol STTC interleaver. Therefore, when longer interleaver delays can be afforded, the $\operatorname{STTC}(4,32)$ is the favored scheme.

\section{BER Performance Versus Delay for NOCC-STTC Systems}

The system delay is mainly determined by the latency introduced by the STTC interleavers, where an entire block of symbols must be stored in the interleaver's buffer before their transmission may commence. Here, the processing delay attributed to the STTC encoding, modulation, and turbo-detection operations has been ignored, although practical systems typically have a processing delay, which allows them to complete their operations just before they have to commence processing the next incoming information block. In general, interactive speech systems can tolerate only limited system delays, which are less than $40 \mathrm{~ms}$.

Let us now review the impact of interleaving length on the NOCC-STTC system's performance by considering, as an example, a TDMA/TDD system, which employs eight uplink and eight downlink slots. Hence, a transmission slot will be available for a specific user after every 16 TDMA slots. Since each $72-\mu$ s burst consists of 144 data symbols, the system delay is $72-\mu$ s for an interleaver length of 144 symbols. In general, we have

$$
\begin{aligned}
\text { System delay }= & {\left[\frac{\text { Interleaver length }}{\text { Data symbols per burst }}\right] } \\
& \times \text { Burst duration } \\
& \times \text { Slots per frame } \\
= & {\left[\frac{\text { Interleaver length }}{144}\right] } \\
& \times 72 \mu \mathrm{s} \times 16
\end{aligned}
$$

if interleaver length $>144$ symbols

corresponding to $4.6,18.4$, and $73.7 \mathrm{~ms}$ for STTC interleaver lengths of 576, 2304, and 9216 symbols, respectively. With the aid of (12), the system delay of the various STTC schemes can be determined, which is illustrated in Fig. 6 in relation to the SNR value required for achieving BER $=10^{-3}$. Specifically, in Fig. 6 it can be observed that, upon increasing the interleaver length, the STTC systems require lower SNR values for achieving a BER of $10^{-3}$. The achievable SNR reduction is marginal for the lower complexity STTC codes, namely, for the $\operatorname{STTC}(4,4)$ and $\operatorname{STTC}(4,8)$ schemes, but becomes more significant for the more powerful STTC codes, such as the STTC $(4,16)$ and $\operatorname{STTC}(4,32)$ arrangements. However, the curves reach a plateau for interleaver lengths higher than 2304 symbols. Therefore, there is no substantial advantage in employing for example a quadruple-delay

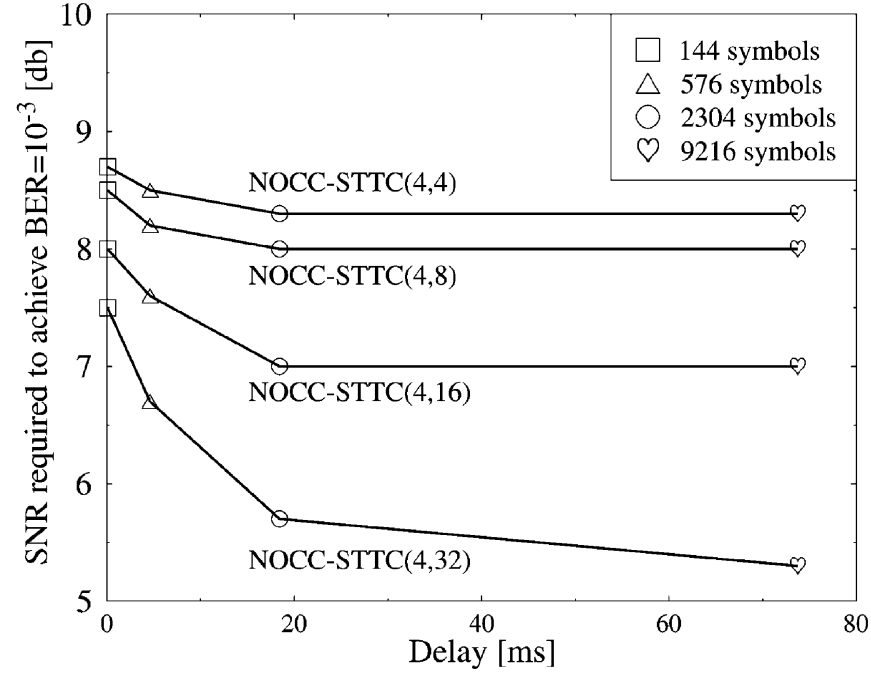

Fig. 6. Performance of the STTC systems that do not employ channel coding, namely, NOCC-STTC $(4,4)$, NOCC-STTC $(4,8)$, NOCC-STTC $(4,16)$, and NOCC-STTC $(4,32)$ schemes after four turbo-detection iterations versus the STTC interleaving delay for the reduced-complexity turbo-detection arrangement, when communicating over a Rayleigh-fading channel exhibiting a two-path, equal-weight, and symbol-spaced CIR associated with a normalized Doppler frequency of $3.3615 \times 10^{-5}$. The effective throughput was 2 bits/s.

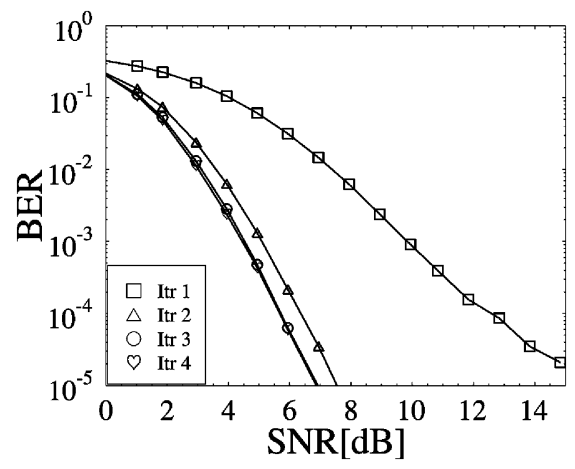

Fig. 7. Performance of the reduced-complexity turbo-detection arrangement in conjunction with the nonchannel-coded STTC $(4,32)$ system using a 2304-symbol STTC interleaver for transmission over a Rayleigh-fading channel exhibiting a five-path, equal-weight, and symbol-spaced CIR associated with a normalized Doppler frequency of $3.3615 \times 10^{-5}$. The effective throughput was 2 bits/s.

9216-symbol interleaver, since the achievable SNR reduction is marginal, while the system delay and storage requirements are significantly increased.

Since interactive speech systems can tolerate only limited system delays, which are typically less than $40 \mathrm{~ms}$, we opt to employ the 2304-symbol interleaver in the following simulations. As mentioned previously, for this particular interleaver length, the $\operatorname{STTC}(4,32)$ scheme is our favored choice. Using these parameters, we investigate the performance of the NOCC-STTC system communicating over channels having a CIR associated with long delay spreads, i.e., transmitting over a Rayleigh-fading channel exhibiting a symbol-spaced, five-path, and equal tap-weight CIR. For this highly dispersive CIR, the F-TD scheme cannot be implemented, since the complexity of the trellis-based channel equalizer alone is already associated with $10^{6}$ transitions per trellis interval. In Fig. 7, it was observed that, despite experiencing long delay spreads, the R-TD was capable of detecting signals transmitted over such highly dispersive channels, while 


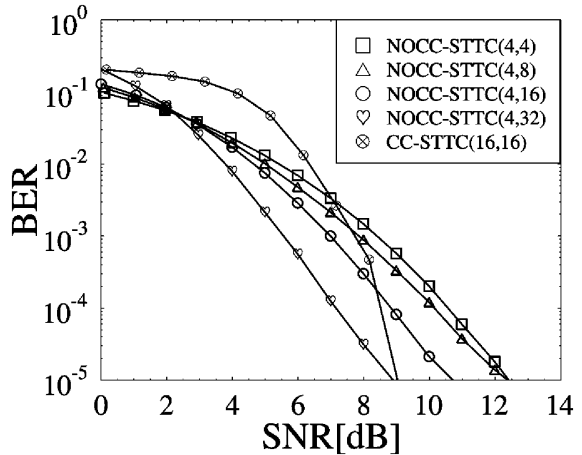

Fig. 8. Performance comparison of the conventional and reduced-complexity turbo-detection schemes in conjunction with the CC-STTC $(4,4)$ scheme using a 2304-symbol STTC interleaver and a 4608-bit channel interleaver for transmission over a Rayleigh-fading channel exhibiting a two-path, equal-weight, and symbol-spaced CIR associated with a normalized Doppler frequency of $3.3615 \times 10^{-5}$. The effective throughput was $1 \mathrm{bits} / \mathrm{s}$.

attaining good BER performances. Assuming that the F-TD scheme could have been simulated and, as a hypothesis, two turbo-detection iterations were required, the R-TD would have achieved a complexity reduction factor of 862 .

\section{BER Performance of the CC-STTC Systems}

Let us now investigate the achievable performance of the R-TD receiver in the context of the more complex CC-STTC schemes utilizing a $R=1 / 2$ and constraint length $K=5$ convolutional-coded four-state quaternary phase-shift keying (QPSK) STTC arrangement employing a 2304-symbol STTC interleaver and a 4608-b channel interleaver for transmission over a Rayleigh-fading channel exhibiting two-path, equal-weight, and symbol-spaced CIR having a normalized Doppler frequency of $3.3165 \times 10^{-5}$. In Fig. 8, it was observed that, after performing three turbo-detection iterations, the R-TD scheme was capable of approaching the performance of the F-TD arrangement, which also utilized three turbo-detection iterations. No further improvements were obtained by performing additional turbo detection iterations. Using (9) and (11), we note that the complexity of the R-TD scheme is a factor of four lower than that of the F-TD scheme. In comparison to the NOCC-STTC system, the CC-STTC scheme is observed to require a significantly lower SNR value for achieving a BER of $10^{-3}$. However, this is attained at the expense of a significant increase in computational complexity and at an effect throughput reduction. Let us now compare the R-TD performance of the CC-STTC scheme to that of the NOCC-STTC systems, while maintaining the same overall throughput for both schemes.

\section{E. Comparison Between the NOCC-STTC and CC-STTC Systems}

In order to ensure that the higher complexity $R=1 / 2$ convolutional-coded STTC scheme possesses the same effective throughput as the NOCC-STTC $(4, n)$ systems, a higher order modulation mode based STTC, such as the STTC $(16,16)$ scheme, was utilized in conjunction with the $R=1 / 2$ convolutional code. Specifically, we compared the R-TD-aided performance of the NOCC-STTC $(4,4)$, NOCCSTTC $(4,8)$, NOCC-STTC $(4,16)$, and NOCC-STTC $(4,32)$ schemes to that of the $R=1 / 2$ convolutional-coded CC-STTC $(16,16)$ scheme using the R-TD technique. In the channel-coded STTC $(16,16)$ scheme, an $R=1 / 2$ and constraint length $K=7$ convolutional code having octal generator polynomials of $G_{0}=133$ and $G_{1}=171$ [13] was utilized. A 2304-symbol random STTC interleaver was also invoked in this system. Here, we have employed a convolutional code that was

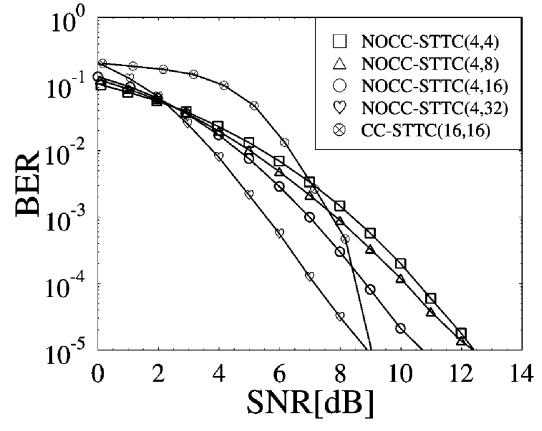

Fig. 9. Comparing the reduced-complexity turbo detection (R-TD) aided performance of the higher complexity $R=1 / 2$ convolutional coded CC-STTC $(16,16)$ scheme to that of lower complexity R-TD NOCC-STTC $(4$, $n$ ) benchmarkers refraining from using channel coding, where we had $n=4,8,16$ and32 STTC trellis states when transmitting over a Rayleigfading channel exhibiting a two-path, equal-weight, and symbol-spaced associated with a normalized Doppler frequency of $3.3615 \times 10^{-5}$. The effective throughput was 2 bits/s.

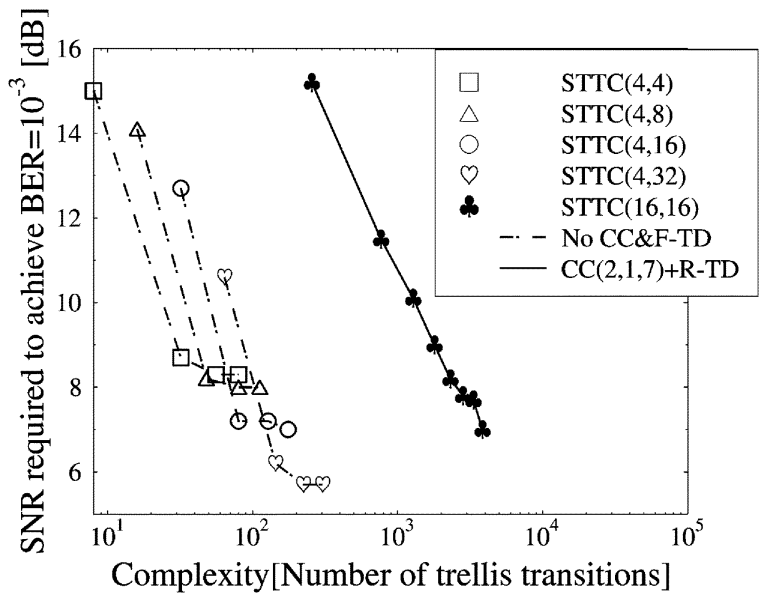

Fig. 10. Comparing the complexity of the reduced complexity turbo detection (R-TD) assisted NOCC-STTC $(4,4)$, NOCC-STTC $(4,8)$, NOCC-STTC $(4,16)$ and NOCC-STTC $(4,32)$ systems with that of the R-TD aided convolutional-coded STTC $(16,16)$ scheme for transmission over a Rayleigh fading channel having a two-path, equal-weight, and symbol-spaced CIR associated with a normalized Doppler frequency of $3.3615 \times 10^{-5}$. In each curve, the complexity increases upon increasing the number of turbo detection iterations. The effective throughput was 2 bits/s.

more complex and powerful than the convolutional code used in the CC-STTC $(4,4)$ scheme in order to protect the transmitted data more effectively. The CC-STTC $(16,16)$ system is more prone to errors, since it utilizes the 16-QAM scheme, which has a more dense, reduced minimum-distance signal constellation than that of 4-PSK.

In Fig. 9, we observed that the $R=1 / 2$ and constraint length $K=7$ convolutional-coded CC-STTC $(16,16)$ scheme employing R-TD and eight turbo-detection iterations was capable of outperforming the NOCC-STTC $(4,4)$ scheme by $0.5 \mathrm{~dB}$ and obtained a similar performance to the NOCC-STTC $(4,8)$ scheme at BER $=10^{-3}$. However, the NOCC-STTC $(4,16)$ and the NOCC-STTC $(4,32)$ arrangements were observed to require 0.8 and $2.3 \mathrm{~dB}$ lower SNR, respectively, than the convolutional-coded CC-STTC $(16,16)$ scheme for achieving the same BER.

Fig. 10 highlights the complexity required by the CC-STTC $(16,16)$ system employing R-TD and that of the $\operatorname{NOCC-STTC}(4, n)$ scheme utilizing the R-TD technique for achieving a BER of $10^{-3}$. It was observed that the $R=1 / 2, K=7$ convolutional-coded CC-STTC $(16,16)$ system invoking eight turbo-detection iterations 
outperforms the NOCC-STTC $(4,4)$ scheme and approaches the performance of the NOCC-STTC $(4,8)$ system using two turbo-detection iterations, although at the cost of incurring a 75 and 50 times higher complexity, respectively. When compared to the NOCC-STTC $(4,16)$ and NOCC-STTC $(4,32)$ schemes using R-TD and performing three turbo-detection iterations, the $R=1 / 2$ and constraint length $K=7$ convolutional-coded CC-STTC $(16,16)$ system invoking eight turbo-detection iterations and using the R-TD technique required a higher SNR value and incurred 11 or 19 times higher computational complexity for achieving BER $=10^{-3}$.

\section{CONCLUSION}

In this paper, we have shown that the principle of I/Q turbo detection, which was originally proposed for single-transmitter and singlereceiver systems [4], [15], can be adapted for employment in STTC systems in order to reduce the complexity of the STTC turbo-detection scheme without sacrificing the achievable performance.

We have characterized the tradeoffs between the achievable performance and the required complexity. In Fig. 5, it was observed that when employing the IQ turbo equalizer scheme, which performed three turbo-detection iterations in the context of the $\operatorname{STTC}(4,4)$, STTC $(4,8)$, STTC $(4,16)$, and $\operatorname{STTC}(4,32)$ systems, the SNR value required for achieving a BER of $10^{-3}$ was comparable to that of the TEQ-CT scheme performing two turbo-detection iterations. Moreover, the TEQ-IQ scheme attained a complexity reduction factor of 9, 6, 4, and 3, respectively. It was also observed in Fig. 5 that, for short interleaver lengths, it was sufficient to employ the lowest complexity STTC $(4,4)$, scheme, since the more computationally complex STTC $(4,16)$ and $\operatorname{STTC}(4,32)$ arrangements yielded modest additional gains. However, for systems where the maximum system delay is not constrained, long-delay STTC interleavers can be used. In conjunction with long interleavers, the $\operatorname{STTC}(4,32)$ scheme is the best choice, since the achievable performance gain becomes more significant. This was further highlighted in Fig. 5(d), where it was observed that the performance of the STTC $(4,32)$ scheme improved upon increasing the interleaver length. From Fig. 6 it was observed that the performance achieved by the schemes utilizing the quadruple-delay 9216-symbol interleaver was only marginally better than that of the systems using the 2304-symbol interleaver, despite incurring a significantly longer system delays of $73.7 \mathrm{~ms}$. Therefore, an interleaver associated with a length of approximately 2304 symbols constitutes an attractive compromise between the achievable performance gain and the system delay imposed.

When comparing the various STTC schemes of the same overall throughput, it was observed from Fig. 9 that the turbo-detected NOCCSTTC $(4,16)$ and NOCC-STTC $(4,32)$ systems were still capable of outperforming the $R=1 / 2$ and constraint length $K=7$ convolutionalcoded CC-STTC $(16,16)$ scheme, while maintaining a BER of $10^{-3}$, even though the CC-STTC $(16,16)$ system incurred approximately 19 or 11 times higher complexity, respectively.

For typical speech and video systems, the target BER may be required between $10^{-4}$ and $10^{-3}$. Therefore, for such schemes the lower complexity turbo-detected NOCC-STTC scheme is the more robust choice. Furthermore, the complexity incurred by these systems is significantly lower than that of the CC-STTC schemes. From these results, it can also be concluded that, for turbo-detected STTC schemes of the same effective throughput, it is better to invest the affordable complexity in implementing higher complexity STTC codes, such as the $\operatorname{STTC}(4,32)$ scheme, than in concatenating a powerful and complex channel encoder with a weak STTC code, such as the STTC $(16,16)$.

\section{ACKNOWLEDGMENT}

The authors would like to thank their colleagues for their contributions.

\section{REFERENCES}

[1] J. Blogh and L. Hanzo, Third-Generation Systems \& Intelligent Wireless Networking: Smart Antennas \& Adaptive Modulation. New York: Wiley/IEEE Press, 2002.

[2] S. Alamouti, "A simple transmit diversity technique for wireless communications," IEEE J. Select. Areas Commun., vol. 16, pp. 1451-1458, Oct. 1998.

[3] V. Tarokh, H. Jafarkhani, and A. Calderbank, "Space-time block codes from orthogonal designs," IEEE Trans. Inform. Theory, vol. 45, pp. 1456-1467, July 1999.

[4] L. Hanzo, T. H. Liew, and B. L. Yeap, Turbo Coding, Turbo Equalization and Space-Time Coding for Transmission over Fading Channels. New York: Wiley/IEEE Press, 2002.

[5] V. Tarokh, N. Seshadri, and A. R. Calderbank, "Space-time codes for high data rate wireless communication: Performance criterion and code construction," IEEE Trans. Inform. Theory, vol. 44, pp. 744-765, Mar. 1998.

[6] B. Hassibi and B. M. Hochwald, "High-rate codes that are linear in space and time," IEEE Trans. Inform. Theory, vol. 48, pp. 1804-1824, July 2002.

[7] A. Hammons and H. E. Gamal, "On the theory of space-time codes for PSK modulation," IEEE Trans. Inform. Theory, vol. 46, pp. 524-542, Mar. 2000.

[8] Z. Safar and K. Liu, "Systematic design of space-time trellis codes for diversity and coding advantages," EURASIP J. Appl. Signal Processing, vol. 2002, pp. 221-235, 2002.

[9] L. Hanzo, M. Münster, B-J. Choi, and T. Keller, OFDM Versus MC-CDMA for Broadband Multi-User Communications, WLANs and Broadcasting. New York: Wiley/IEEE Press, 2003.

[10] C. Douillard, A. Picart, M. Jézéquel, P. Didier, C. Berrou, and A. Glavieux, "Iterative correction of intersymbol interference: Turbo-equalization," Eur. Trans. Commun., vol. 6, pp. 507-511, 1995.

[11] G. Bauch and N. Al-Dhahir, "Reduced-complexity space-time turbo-equalization for frequency-selective MIMO channels," IEEE Trans. Wireless Commun., vol. 1, pp. 819-828, Oct. 2002.

[12] B. L. Yeap, T. H. Liew, and L. Hanzo, Proc. Turbo Equalization of Serially Concatenated Systematic Convolutional Codes and Systematic Space Time Trellis Codes, vol. 2, Rhodes, Greece, May 2001, pp. $1464-1468$.

[13] "Digital video broadcast (DVB): Framing structure, channel coding and modulation for 11/12 GHz satellite services," Eur. Telecommun. Standardiation Inst. (ETSI), 1997.

[14] A. Glavieux, C. Laot, and J. Labat, "Turbo equalization over a frequency selective channel," in Proc. Int. Symp. Turbo Codes Related Topics, Brest, France, Sept. 1997, pp. 96-102.

[15] B. L. Yeap, C. H. Wong, and L. Hanzo, "Reduced complexity in-phase/quadrature-phase turbo equalization," IEEE Trans. Wireless Commun., vol. 2, pp. 2-10, Jan. 2003.

[16] C. Tidestav, M. Sternad, and A. Ahlén, "Reuse within a cell—Interference rejection or multiuser detection," IEEE Trans. Commun., vol. 47, pp. 1511-1522, Oct. 1999.

[17] W. Koch and A. Baier, "Optimum and sub-optimum detection of coded data disturbed by time-varying inter-symbol interference," in Proc. IEEE Global Telecommunications Conf., San Diego, CA, Dec. 1990 pp. $1679-1684$.

[18] J. A. Erfanian, S. Pasupathy, and G. Gulak, "Reduced complexity symbol detectors with parallel structures for ISI channels," IEEE Trans. Commun., vol. 42, pp. 1661-1671, Feb./Mar./Apr. 1994. 